\title{
An Evaluation of Voluntary Varicella Vaccination Coverage in Zhejiang Province, East China
}

\author{
Yu Hu, Yaping Chen, Bing Zhang and Qian Li * \\ Institute of Immunization and Prevention, Zhejiang Provincial Center for Disease Control and Prevention, \\ Hangzhou 310051, China; husix@163.com (Y.H.); ypchen@cdc.zj.cn (Y.C.); bzhang@cdc.zj.cn (B.Z.) \\ * Correspondence: qianli@cdc.zj.cn; Tel.: +86-87115167
}

Academic Editor: Paul B. Tchounwou

Received: 11 April 2016; Accepted: 31 May 2016; Published: 3 June 2016

\begin{abstract}
Background: In 2014 a 2-doses varicella vaccine (VarV) schedule was recommended by the Zhejiang Provincial Center for Disease Control and Prevention. We aimed to assess the coverage of the 1st dose of $\operatorname{VarV}\left(\operatorname{VarV}_{1}\right)$ and the 2 nd dose of $\operatorname{VarV}\left(\operatorname{VarV}_{2}\right)$ among children aged 2-6 years through the Zhejiang Provincial Immunization Information System (ZJIIS) and to explore the determinants associated with the VarV coverage. Methods: Children aged 2-6 years (born from 1 January 2009 to 31 December 2013) registered in ZJIIS were enrolled. Anonymized individual records of target children were extracted from the ZJIIS database on 1 January 2016, including their VarV and (measles-containing vaccine) $M C V$ vaccination information. The $\operatorname{VarV}_{1}$ and $\operatorname{Var} V_{2}$ coverage rates were evaluated for each birth cohorts. The coverage of VarV also was estimated among strata defined by cities, gender and immigration status. We also evaluated the difference in coverage between VarV and MCV. Results: A total of 3,028,222 children aged 2-6 years were enrolled. The coverage of VarV $V_{1}$ ranged from $84.8 \%$ to $87.9 \%$ in the $2009-2013$ birth cohorts, while the coverage of $\operatorname{VarV}_{2}$ increased from $31.8 \%$ for the 2009 birth cohort to $48.7 \%$ for the 2011 birth cohort. Higher coverage rates for both $\operatorname{VarV}_{1}$ and $\operatorname{VarV}_{2}$ were observed among resident children in relevant birth cohorts. The coverage rates of $\operatorname{VarV}_{1}$ and $\operatorname{VarV}_{2}$ were lower than those for the 1st and 2nd dose of MCV, which were above $95 \%$. The proportion of children who were vaccinated with $\operatorname{VarV}_{1}$ at the recommended age increased from $34.6 \%$ for the 2009 birth cohort to $75.2 \%$ for the 2013 birth cohort, while the proportion of children who were vaccinated with $\operatorname{VarV}_{2}$ at the recommended age increased from $19.7 \%$ for the 2009 birth cohort to $48.7 \%$ for the 2011 birth cohort. Conclusions: Our study showed a rapid increasing $\operatorname{VarV}_{2}$ coverage of children, indicating a growing acceptance of the 2-doses VarV schedule among children's caregivers and physicians after the new recommendation released. We highlighted the necessity for a 2-doses VarV vaccination school-entry requirement to achieve the high coverage of $>90 \%$ and to eliminate disparities in coverage among sub-populations. We also recommended continuous monitoring of the VarV coverage via ZJIIS over time.
\end{abstract}

Keywords: varicella vaccine; immunization information system; coverage; birth cohort

\section{Introduction}

Since 1998, varicella vaccine (VarV) has been licensed in Zhejiang Province, which is a developed province with a large population of 80 million located in East China. The 1-dose of VarV schedule for routine use in children aged $\geqslant 12$ months was recommended by Zhejiang Provincial Center for Disease Control and Prevention (ZJCDC) since the approval of VarV. VarV is a category II (parent-pay) vaccine in China and the vaccination is voluntary. Since the schedule of 1-dose of VarV was recommended, VarV coverage increased rapidly and a substantial decrease in the incidence of varicella disease, varicella-related morbidity and mortality, and health care costs had been observed. Outbreaks of varicella, however, continued to occur in kindergartens or primary schools among children with high 
coverage of 1-dose VarV in Zhejiang Province. Some studies reported that approximately $15 \%$ of VarV recipients did not achieve protective levels of antibody and VarV induced immunity waned with time [1]. Besides, a large randomized trial indicated that the 2-doses VarV schedule was significantly more effective than the 1-dose VarV schedule [2]. In 2014, ZJCDC updated the recommendation regarding the use of VarV for varicella prevention. The latest recommendation includes a 3-pronged approach: (1) a routine 2-doses $\operatorname{VarV}$ schedule, with the 1st dose of $\operatorname{VarV}\left(\operatorname{VarV}_{1}\right)$ administrated at 12-15 months of age and the $2 \mathrm{nd}$ dose of $\operatorname{VarV}\left(\operatorname{VarV}_{2}\right)$ administrated at 3-4 years of age; (2) a $\operatorname{VarV}_{2}$ catch-up vaccination for children and adolescents aged $\leqslant 12$ years who received $\operatorname{Var} V_{1}$ previously and without a history of varicella disease; ( 3 ) all persons aged $\geqslant 13$ years without any evidence of immunity should be given 2 doses of VarV. According to the new recommendation, a minimum interval of 28 days between two doses of VarV is required.

At present, it is important to understand and continuously monitor the coverage of VarV among eligible age groups to guide future policy and intervention design. However, VarV coverage data of eligible children is not available as until now all the category II vaccines have not been included in the provincial level vaccination surveys. Therefore, little is known about the coverage of VarV by demographic characteristics such as gender or migration status. In fact, understanding of the coverage of VarV by these characteristics can provide valuable information for developing interventions tailored to subgroups with lower coverage.

The main objectives of this study included: (1) evaluating the coverage of VarV and exploring its determinants for children aged 2-6 years, and assessing the proportion of children who were vaccinated at the recommended age; (2) comparing the coverage of VarV with the coverage of measles containing vaccine (MCV), which had been included in the national immunization program (NIP).

\section{Methods}

\subsection{Data Source}

Data on VarV coverage were obtained from Zhejiang Provincial Immunization Information System (ZJIIS). ZJIIS, also known as the immunization registries, is a computerized, population-based system containing demographic and vaccination data for children aged $<7$ years living in Zhejiang Province since 2004 [3]. All vaccination clinics in Zhejiang Province must participate in ZJIIS. ZJIIS includes a client software deployed at each vaccination clinic and a database deployed in ZJCDC. The ZJIIS database consolidates data from different vaccination clinics through the Internet in real time and provides a tool for supporting effective vaccination strategies at the provider and program management level. Once any immunization clinic in Zhejiang Province is visited for the first time, children will be registered in ZJIIS with a unique identifier. Historical immunization information for migrant children is also included. Demographic information (such as name, date of birth, gender, address, phone number, immigration status) and vaccination records are collected by ZJIIS and these information will be updated in a day if there is any change.

Children aged 2-6 years (born from 1 January 2009 to 31 December 2013) and registered in ZJIIS were enrolled in this study. Appropriately anonymized individual records of target children were extracted from the ZJIIS database on 1 January 2016, including the VarV and MCV vaccination information. Children who were designated in ZJIIS as permanently inactive (i.e., deceased) or "moved or gone elsewhere" were excluded.

\subsection{Definitions}

The $\operatorname{Var} V_{1}$ coverage was defined as the proportion of children who received $\operatorname{Var} V_{1}$, while the $\operatorname{Var}_{2}$ coverage was defined as the proportion of children with $\operatorname{Var} V_{1}$ who received $\operatorname{Var} V_{2}$. Valid doses of VarV were defined as $\operatorname{VarV}_{1}$ administered no earlier than 4 days prior to the first birthday, $\operatorname{Var} V_{2}$ administered at least 28 days after $\operatorname{VarV}_{1}$, and either dose administered on the same day as or $\geqslant 4$ weeks 
after any other live attenuated vaccine. All the invalid doses were considered as "unvaccinated" and were excluded from analysis.

The data were analyzed by birth cohort to evaluate the coverage of VarV at population or geography (by city) level, and for comparing the coverage of VarV and MCV. 12-month wide cohort was used for analysis among different cities, gender or immigration status. These cohorts were children born between 1 January, and 31 December 2009 for the 2009 birth cohort; children born between 1 January and 31 December 2010 for the 2010 birth cohort; children born between 1 January and 31 December 2011 for the 2011 birth cohort; children born between 1 January and 31 December 2012 for the 2012 birth cohort; children born between 1 January and 31 December 2013 for the 2013 birth cohort. The coverage of $\operatorname{VarV}_{1}$ was calculated for all the five cohorts while the coverage of $\operatorname{VarV}_{2}$ was calculated only for the 2009, 2010 and 2011 cohorts. Immigration status of children was classified as resident and migrant in this study. Migrant children included children from other provinces or from other cities of Zhejiang Province or from abroad as they were categorized in ZJIIS.

\subsection{Analysis Strategy}

The coverage rates of $\operatorname{VarV}_{1}$ and $\operatorname{VarV}_{2}$ of the relevant birth cohorts were calculated separately, as well as the proportion of children who were vaccinated at the recommended age 12-15 months for the $\operatorname{VarV}_{1}$ and 3-4 years for the $\operatorname{VarV}_{2}$. The VarV coverage was estimated among strata defined by cities, gender and immigration status. We evaluated separately the coverage of $\operatorname{Var}_{1}$ and $\operatorname{Var} V_{2}$ in parallel to the coverage of the 1 st and the 2 nd dose MCV, which were scheduled at 8 months of age and 18 months of age, respectively. A $\chi^{2}$ test for trends in different birth cohorts was performed to evaluate whether there was a significant increase in coverage of VarV over time. We adopted the $\chi^{2}$ test to examine whether the coverage of VarV was significantly different across subgroup strata defined by gender or immigration status. We compared the coverage of MCV and VarV and evaluated the difference with the $\chi^{2}$ test. We performed all analysis with SPSS version 13.0 (SPSS Inc., Chicago, IL, USA) and at a significance level of 0.05 .

\subsection{Ethical Considerations}

This study was approved by the Ethical Review Board of ZJCDC (No. T-043-R). All the data were anonymous when we exported them from ZJIIS and kept confidential without individual identifiers.

\section{Results}

\subsection{Overall Coverage of VarV}

A total of 3,028,222 children aged 2-6 years (born from 1 January 2009 to 31 December 2013) were enrolled in this study, of which $50.5 \%(1,527,818)$ were male and $60.0 \%(1,817,679)$ were migrant children. The $\operatorname{VarV}_{1}$ coverage for the 2009 birth cohort to the 2013 cohort was $84.8 \%, 86.8 \%, 88.6 \%$, $88.5 \%, 87.9 \%$, respectively. Across different birth cohorts, a significant increase in the coverage of $\operatorname{VarV}_{1}$ over time was observed in both the provincial and city level $(p<0.01)$. There were significant disparities in the coverage of $\operatorname{VarV}_{1}$ across the 10 cities for all the five birth cohorts. The coverage rates of $\operatorname{VarV}_{1}$ of all five cohorts in Wenzhou (WZ), Jiaxing (JX), Shaoxing (SX), and Jinhua (JH) were lower than the average rate of Zhejiang Province. The coverage rates of $\operatorname{VarV}_{1}$ of the 2012 birth cohort in WZ, JX, SX, JH, Quzhou (QZ), and Zhoushan (ZS) were lower than the average rate of Zhejiang Province. The coverage rates of $\operatorname{VarV}_{1}$ of the 2013 birth cohort in WZ, JX, JH, QZ, ZS were lower than the average rate of Zhejiang Province (Table 1). 
A rapid increase of the $\operatorname{VarV}_{2}$ coverage was observed after the new VarV schedule was recommended in 2014. The $\operatorname{VarV}_{2}$ coverage increased significantly from $31.8 \%$ for the 2009 birth cohort to $48.7 \%$ for the 2011 birth cohort. Across the three birth cohorts, a significant increase in the $\operatorname{VarV}_{2}$ coverage over time was observed at both the provincial and city level $(p<0.01)$. There were significant disparities in $\operatorname{VarV}_{2}$ coverage across the 10 cities for the three birth cohorts. The coverage rates of $\operatorname{VarV}_{2}$ of the 2009 birth cohort in $\mathrm{WZ}, \mathrm{SX}, \mathrm{JH}, \mathrm{ZS}$ were lower than the average rate of Zhejiang Province. The $\operatorname{VarV}_{2}$ coverage rates of the 2010 birth cohort in WZ, SX, JH, QZ, ZS were lower than the average rate of Zhejiang Province. The $\operatorname{VarV}_{1}$ coverage rates of the 2011 birth cohort in WZ, JH, QZ, ZS, and Taizhou (TZ) were lower than the average rate of Zhejiang Province (Table 2).

\subsection{VarV Coverage across Subgroups}

The $\operatorname{VarV}_{1}$ coverage was $87.6 \%$ for males and $87.2 \%$ for females in the 2009-2013 birth cohorts, and the $\operatorname{VarV}_{2}$ coverage was $38.3 \%$ for males and 37.3\% for females in in the 2009-2011 birth cohorts. There was no significant difference in the VarV coverage between strata defined by gender in the 2009-2013 birth cohorts for the 1st dose, as for the 2nd dose (Table 3).

The $\operatorname{VarV}_{1}$ coverage was $96.3 \%$ for resident children and $81.5 \%$ for migrant children in the 2009-2013 birth cohorts, and the $\operatorname{VarV}_{2}$ coverage was $52.7 \%$ for resident children and $28.2 \%$ for migrant children in the 2009-2011 birth cohorts. There was significant difference in $\operatorname{VarV}_{1}$ and $\operatorname{VarV}_{2}$ coverage between strata defined by the immigration status of children. Higher coverage was observed in resident children in the 2009-2013 birth cohorts for $\operatorname{VarV}_{1}$, and similar pattern was also observed for $\operatorname{VarV}_{2}$ in the 2009-2011 birth cohorts (Table 4).

\subsection{Coverage Difference between VarV and $M C V$}

The $\operatorname{VarV}_{1}$ coverage increased from $84.8 \%$ for the 2009 birth cohort to $87.9 \%$ for the 2013 birth cohort, and it approached the coverage of the 1 st dose of MCV (96.3\%-97.8\%). The $\operatorname{VarV}_{2}$ coverage of in the 2009-2011 birth cohorts was relatively consistent and it was significant lower than the 2nd dose coverage of MCV (95.5\%-96.2\%) (Table 5). 
Table 1. Vaccination coverage of $\operatorname{VarV}_{1}$ of children aged 4-6 years in Zhejiang province, by city.

\begin{tabular}{|c|c|c|c|c|c|c|c|c|c|c|c|}
\hline \multirow{3}{*}{ City \# } & \multicolumn{10}{|c|}{ Coverage of $\operatorname{VarV}_{1}$} & \multirow{3}{*}{$p^{\S}$} \\
\hline & \multicolumn{2}{|c|}{ Birth Cohort 2009* } & \multicolumn{2}{|c|}{ Birth Cohort 2010 * } & \multicolumn{2}{|c|}{ Birth Cohort 2011 * } & \multicolumn{2}{|c|}{ Birth Cohort 2012 * } & \multicolumn{2}{|c|}{ Birth Cohort 2013 * } & \\
\hline & $n / N$ & $\%$ & $n / N$ & $\%$ & $n / N$ & $\%$ & $n / N$ & $\%$ & $n / N$ & $\%$ & \\
\hline $\mathrm{HZ}$ & $78,821 / 87,784$ & 89.8 & $85,349 / 94,079$ & 90.7 & $97,873 / 106,238$ & 92.1 & $111,272 / 121,288$ & 91.7 & $104,740 / 115,606$ & 90.6 & $<0.01$ \\
\hline NB & $80,385 / 87,082$ & 92.3 & $85,937 / 91,708$ & 93.7 & $95,951 / 100,381$ & 95.6 & $103,641 / 108,063$ & 95.9 & $98,724 / 102,965$ & 95.9 & $<0.01$ \\
\hline WZ & $80,844 / 98,593$ & 82.0 & $84,510 / 99,525$ & 84.9 & $90,824 / 107,116$ & 84.8 & $103,157 / 124,049$ & 83.2 & $96,712 / 118,519$ & 81.6 & $<0.01$ \\
\hline$J X$ & $30,922 / 39,661$ & 78.0 & $36,063 / 45,009$ & 80.1 & $38,802 / 46,667$ & 83.1 & $47,190 / 56,486$ & 83.5 & $44,869 / 53,859$ & 83.3 & $<0.01$ \\
\hline SX & $27,947 / 36,115$ & 77.4 & $28,910 / 36,808$ & 78.5 & $34,795 / 41,817$ & 83.2 & $41,035 / 46,788$ & 87.7 & $40,444 / 45,639$ & 88.6 & $<0.01$ \\
\hline $\mathrm{JH}$ & $56,105 / 73,589$ & 76.2 & $60,180 / 74,791$ & 80.5 & $67,601 / 80,547$ & 83.9 & $79,048 / 92,846$ & 85.1 & $76,178 / 89,505$ & 85.1 & $<0.01$ \\
\hline $\mathrm{QZ}$ & $15,509 / 18,220$ & 85.1 & $13,909 / 16,055$ & 86.6 & $16,661 / 19,090$ & 87.3 & $19,462 / 22,368$ & 87.0 & $18,398 / 21,217$ & 86.7 & $<0.01$ \\
\hline ZS & $6094 / 7102$ & 85.8 & $6257 / 7298$ & 85.7 & $6572 / 7655$ & 85.9 & $7591 / 9034$ & 84.0 & $7062 / 8393$ & 84.1 & $<0.01$ \\
\hline $\mathrm{TZ}$ & $60,269 / 67,403$ & 89.4 & $61,264 / 67,565$ & 90.7 & $65,859 / 72,124$ & 91.3 & $73,101 / 81,595$ & 89.6 & $68,500 / 77248$ & 88.7 & $<0.01$ \\
\hline LS & $16,917 / 19,612$ & 86.3 & $15,423 / 17,609$ & 87.6 & $19,279 / 21,329$ & 90.4 & $20,470 / 22,557$ & 90.7 & $19,450 / 21,627$ & 89.9 & $<0.01$ \\
\hline Total & $453,813 / 535,161$ & 84.8 & $477,802 / 550,447$ & 86.8 & $534,217 / 602,964$ & 88.6 & $605,967 / 685,074$ & 88.5 & $575,075 / 654,576$ & 87.9 & $<0.01$ \\
\hline
\end{tabular}

${ }^{\#}$ HZ: HangZhou; NB: NingBo; WZ: WenZhou; JX: JiaXing; SX: ShaoXing; JH: JinHua; QZ: QuZhou; ZS: ZhouShan; LS: LiShui; TZ: TaiZhou; * Difference in coverage among 10 cities was significant; $\S^{\S}$ Two-tailed $p$-value of the $\chi^{2}$ test.

Table 2. Vaccination coverage of VarV2 of children aged 4-6 years in Zhejiang province, by city.

\begin{tabular}{|c|c|c|c|c|c|c|c|}
\hline \multirow{3}{*}{ City $\#$} & \multicolumn{6}{|c|}{ Coverage of $\operatorname{VarV}_{2}$} & \multirow{3}{*}{$p^{\S}$} \\
\hline & \multicolumn{2}{|c|}{ Birth Cohort 2009* } & \multicolumn{2}{|c|}{ Birth Cohort $2010 *$} & \multicolumn{2}{|c|}{ Birth Cohort 2011* } & \\
\hline & $n / N$ & $\%$ & $n / N$ & $\%$ & $n / N$ & $\%$ & \\
\hline $\mathrm{HZ}$ & $32,701 / 87,784$ & 37.3 & $39,378 / 94,079$ & 41.9 & $58,038 / 106,238$ & 54.6 & $<0.01$ \\
\hline NB & $52,631 / 87,082$ & 60.4 & $42,102 / 91,708$ & 45.9 & $75,853 / 100,381$ & 75.6 & $<0.01$ \\
\hline WZ & $13,625 / 98,593$ & 13.8 & $16,375 / 99,525$ & 16.5 & $27,300 / 107,116$ & 25.5 & $<0.01$ \\
\hline JX & $14,237 / 39,661$ & 35.9 & $15,334 / 45,009$ & 34.1 & $22,731 / 46,667$ & 48.7 & $<0.01$ \\
\hline SX & $7492 / 36115$ & 20.7 & $7643 / 36,808$ & 20.8 & $21,674 / 41,817$ & 51.8 & $<0.01$ \\
\hline $\mathrm{JH}$ & $12,808 / 73,589$ & 17.4 & $21,307 / 74,791$ & 28.5 & $33,361 / 80,547$ & 41.4 & $<0.01$ \\
\hline QZ & $6142 / 18,220$ & 33.7 & $4981 / 16,055$ & 31.0 & $8575 / 19,090$ & 44.9 & $<0.01$ \\
\hline ZS & $582 / 7102$ & 8.2 & $1392 / 7298$ & 19.1 & $3268 / 7655$ & 42.7 & $<0.01$ \\
\hline $\mathrm{TZ}$ & $23,513 / 67,403$ & 34.9 & $23,342 / 67,565$ & 34.5 & $31,867 / 72,124$ & 44.2 & $<0.01$ \\
\hline LS & $6348 / 19,612$ & 32.4 & $6018 / 17,609$ & 34.2 & $11,161 / 21,329$ & 52.3 & $<0.01$ \\
\hline Total & $170,079 / 535,161$ & 31.8 & $177,872 / 550,447$ & 32.3 & $293,828 / 602,964$ & 48.7 & $<0.01$ \\
\hline
\end{tabular}

\# HZ: HangZhou; NB: NingBo; WZ: WenZhou; JX: JiaXing; SX: ShaoXing; JH: JinHua; QZ: QuZhou; ZS: ZhouShan; LS: LiShui; TZ: TaiZhou; * Difference in coverage among 10 cities was significant; ${ }^{\S}$ Two-tailed $p$-value of the $\chi^{2}$ test. 
Table 3. Vaccination coverage of VarV for children born from 2009 to 2013 in Zhejiang province, by gender.

\begin{tabular}{|c|c|c|c|c|c|c|c|c|c|c|}
\hline \multirow{3}{*}{$\begin{array}{l}\text { Birth } \\
\text { Cohort }\end{array}$} & \multicolumn{5}{|c|}{ Coverage of $\operatorname{VarV}_{1}$} & \multicolumn{5}{|c|}{ Coverage of $\operatorname{VarV}_{2}$} \\
\hline & \multicolumn{2}{|l|}{ Male } & \multicolumn{2}{|l|}{ Female } & \multirow{2}{*}{$p^{*}$} & \multicolumn{2}{|l|}{ Male } & \multicolumn{2}{|l|}{ Female } & \multirow{2}{*}{$p^{*}$} \\
\hline & $n / N$ & $\%$ & $n / N$ & $\%$ & & $n / N$ & $\%$ & $n / N$ & $\%$ & \\
\hline 2009 & $228,808 / 269,186$ & 85.0 & $225,008 / 265,975$ & 84.6 & $>0.05$ & $86,678 / 269,186$ & 32.2 & $83,503 / 265,975$ & 31.4 & $>0.05$ \\
\hline 2010 & $243,835 / 279,627$ & 87.2 & $233,953 / 270,820$ & 86.4 & $>0.05$ & $91,158 / 279,627$ & 32.6 & $86,636 / 270,820$ & 32.0 & $>0.05$ \\
\hline 2011 & $271,539 / 305,100$ & 89.0 & $262,687 / 297,864$ & 88.2 & $>0.05$ & $149,194 / 305,100$ & 48.9 & $144,450 / 297,864$ & 48.5 & $>0.05$ \\
\hline 2012 & $305,485 / 345,962$ & 88.3 & $300,806 / 339,112$ & 88.7 & $>0.05$ & - & - & - & - & - \\
\hline 2013 & $288,917 / 327,942$ & 88.1 & $286,455 / 326,633$ & 87.7 & $>0.05$ & - & - & - & - & - \\
\hline Total & $1,338,584 / 1,527,818$ & 87.6 & $1,308,909 / 1,500,404$ & 87.2 & $>0.05$ & $327,030 / 853,913$ & 38.3 & $314,589 / 834,659$ & 37.7 & $>0.05$ \\
\hline
\end{tabular}

Table 4. Vaccination coverage of VarV for children born from 2009 to 2013 in Zhejiang province, by immigration status.

\begin{tabular}{|c|c|c|c|c|c|c|c|c|c|c|}
\hline \multirow{3}{*}{$\begin{array}{l}\text { Birth } \\
\text { Cohort }\end{array}$} & \multicolumn{5}{|c|}{ Coverage of $\operatorname{VarV}_{1}$} & \multicolumn{5}{|c|}{ Coverage of $\operatorname{VarV}_{1}$} \\
\hline & \multicolumn{2}{|c|}{ Resident Children } & \multicolumn{2}{|c|}{ Migrant Children } & \multirow{2}{*}{$p^{*}$} & \multicolumn{2}{|c|}{ Resident Children } & \multicolumn{2}{|c|}{ Migrant Children } & \multirow{2}{*}{$p^{*}$} \\
\hline & $n / N$ & $\%$ & $n / N$ & $\%$ & & $n / N$ & $\%$ & $n / N$ & $\%$ & \\
\hline 2009 & $199,470 / 217,625$ & 91.7 & $254,347 / 317,536$ & 80.1 & $<0.01$ & $98,418 / 217,625$ & 45.2 & $71,763 / 317,536$ & 22.6 & $<0.01$ \\
\hline 2010 & $208,419 / 219,120$ & 95.1 & $269,369 / 331,327$ & 81.3 & $<0.01$ & $100,927 / 219,120$ & 46.1 & $76,868 / 331,327$ & 23.2 & $<0.01$ \\
\hline 2011 & $236,217 / 241,302$ & 97.9 & $298,010 / 361,662$ & 82.4 & $<0.01$ & $157,659 / 241,302$ & 65.3 & $135,985 / 361,662$ & 37.6 & $<0.01$ \\
\hline 2012 & $267,962 / 271,974$ & 98.5 & $338,329 / 413,100$ & 81.9 & $<0.01$ & - & - & - & - & - \\
\hline 2013 & $253,823 / 260,521$ & 97.4 & $321,548 / 394,054$ & 81.6 & $<0.01$ & - & - & - & - & - \\
\hline Total & $1,165,891 / 1,210,542$ & 96.3 & $1,481,602 / 1,817,679$ & 81.5 & $<0.01$ & $357,003 / 678,047$ & 52.7 & $284,616 / 1,010,525$ & 28.2 & $<0.01$ \\
\hline
\end{tabular}

* Two-tailed $p$-value of the $\chi^{2}$ test.

Table 5. Comparative assessment of vaccination coverage of VarV and MCV for children born from 2009 to 2013 in Zhejiang province.

\begin{tabular}{ccccccc}
\hline \multirow{2}{*}{$\begin{array}{c}\text { Birth } \\
\text { Cohort }\end{array}$} & \multicolumn{3}{c}{ Coverage of the 1st Dose (\%) } & \multicolumn{3}{c}{ Coverage of the 2nd Dose (\%) } \\
\cline { 2 - 7 } & VarV & MCV & $\boldsymbol{p}^{*}$ & VarV & MCV & $\boldsymbol{p}^{*}$ \\
\hline 2009 & 84.8 & 96.3 & $<0.01$ & 31.8 & 95.5 & $<0.01$ \\
2010 & 86.8 & 96.3 & $<0.01$ & 32.3 & 96.2 & $<0.01$ \\
2011 & 88.6 & 97.8 & $<0.01$ & 48.7 & 96.4 & $<0.01$ \\
2012 & 88.5 & 97.4 & $<0.01$ & - & - & - \\
2013 & 87.9 & 96.8 & $<0.01$ & - & - & - \\
Total & 87.4 & 97.0 & $<0.01$ & 38.0 & 96.0 & $<0.01$ \\
\hline \multicolumn{7}{c}{${ }^{*}$ Two-tailed $p$-value of the $\chi^{2}$ test. } \\
\end{tabular}




\subsection{Age of Vaccinated Children}

The proportions of children who received $\operatorname{VarV}_{1}$ at the recommended age at 12-15 months for the 2009 birth cohort to the 2013 cohort were $34.6 \%, 44.3 \%, 57.6 \%, 68.5 \%, 75.2 \%$, respectively (Figure 1). The proportion of children who received $\operatorname{VarV}_{2}$ at the recommended age at 36-48 months (3-4 years) for the 2009 birth cohort to the 2011 cohort 2011 was 19.7\%, 23.4\%, 48.7\%, respectively (Figure 2).

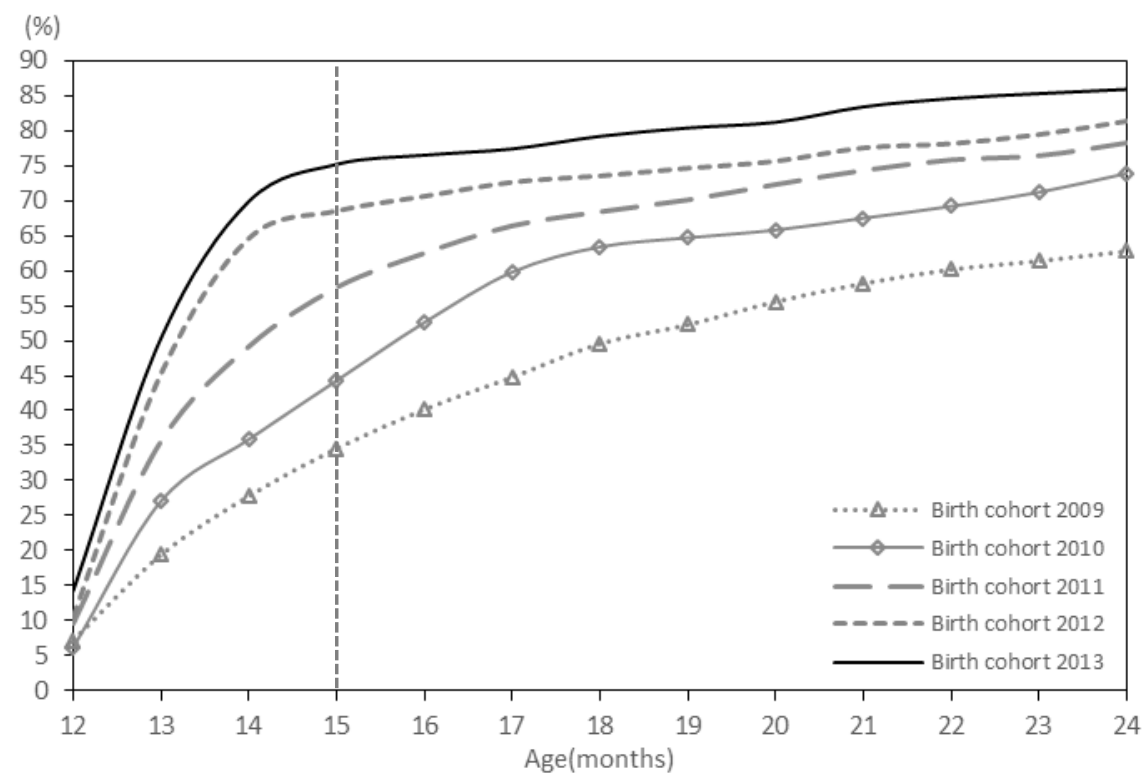

Figure 1. The coverage rate of $\operatorname{VarV}_{1}$ for children aged 2-6 years.

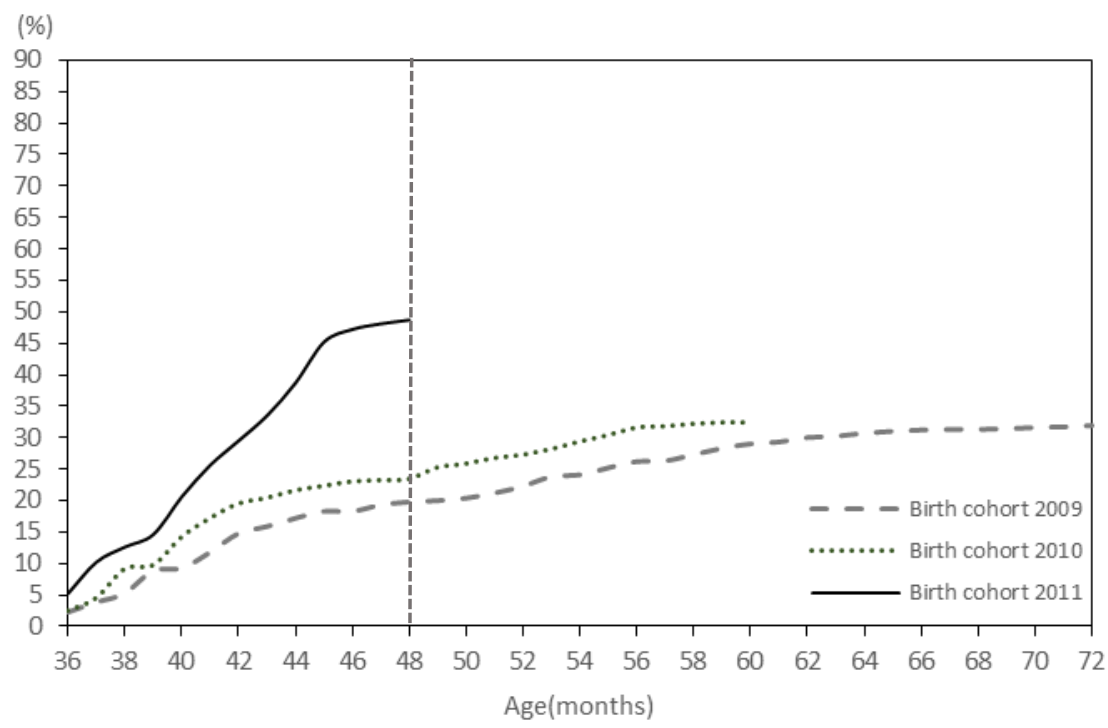

Figure 2. The coverage rate of $\operatorname{VarV}_{2}$ for children aged 4-6 years.

\section{Discussion}

Since the introduction of the 1-dose schedule of $\operatorname{VarV}$, the $\operatorname{VarV}_{1}$ coverage increased significantly from the 2009 birth cohort to the 2013 birth cohort, approaching the goal of $>90 \%$ for China's NIP vaccines, but it was still lower than the VarV1 coverage in the USA [4], where the $\operatorname{VarV}_{1}$ coverage was $96.2 \%$ (95\%CI: $96.2 \%-96.3 \%$ ) for children aged $\geqslant 4$ years in 2015 . The high $\operatorname{VarV}_{1}$ coverage in the USA 
is mainly attributed to the 1-dose varicella vaccination school-entry requirement in place since 1999, which was changed to a 2-doses varicella vaccination requirement in 2006 [5].

Although the population-based incidence of varicella was not available due to the fact that until now varicella is not a notifiable disease in China, the national public health emergencies reporting system shows a steady decrease in the outbreaks of varicella in children aged $\leqslant 6$ years in recent years.

Achieving a high 2-doses VarV coverage is critical for preventing varicella as the incremental vaccine effectiveness of 2-doses versus 1-dose of VarV was estimated as 63.6\% [6]. Furthermore, even among the children who received $\operatorname{VarV}_{1}$ before, receipt the $\operatorname{VarV}_{2}$ also is important as the antibody level induced by $\operatorname{VarV}_{1}$ may wane with time since administration of $\operatorname{VarV}$, which increases the risk of breakthrough varicella [7]. In this study, the $\operatorname{VarV}_{2}$ coverage was increasing rapidly. The results indicated the new recommendation of VarV vaccination was being quickly and widely accepted by physicians and caregivers and the $\operatorname{VarV}_{2}$ coverage for the 2011 birth cohort was much higher than that for the older children. These findings also demonstrated the relative success of the introduction of the 2-doses VarV schedule. However, the $\operatorname{VarV}_{2}$ coverage was still lower than that of $\operatorname{VarV}_{1}$, which potentially places unvaccinated children at risk for breakthrough varicella. Breakthrough varicella had been demonstrated as an impact factor in several prolonged outbreaks of varicella in high population intensity settings like schools or kindergartens, because breakthrough varicella is highly infectious and difficult to recognize [8].

Regional disparities in coverage were observed for both $\operatorname{VarV}_{1}$ and $\operatorname{VarV}_{2}$. Children from HZ, $\mathrm{NB}, \mathrm{TZ}$, LS had a significantly higher $\operatorname{VarV}_{1}$ coverage than that of children from other cities. Aside for the reason that VarV was a category II vaccine, this finding might be associated with the school-entry $\operatorname{VarV}_{1}$ vaccination requirement in these four cities, in addition to the school-entry requirement only for NIP vaccine in Zhejiang Province. We also found that $\mathrm{HZ}, \mathrm{NB}, \mathrm{JX}$, LS had a higher $\operatorname{VarV}_{2}$ coverage than other cities. The $\operatorname{VarV}_{2}$ catch-up campaigns targeting the children aged 4-6 years with $\operatorname{VarV}_{1}$ which were conducted in 2015 in these four cities might explain the higher coverage of $\operatorname{VarV}_{2}$.

Children's age remained a determinant of the $\operatorname{Var}_{2}$ coverage, with a higher coverage seen for younger children in this study. The lower $\operatorname{VarV}_{2}$ coverage among older children might represent the failure to be vaccinated through a catch-up schedule. Another plausible explanation was that some of the older children with $\operatorname{VarV}_{1}$ might have had breakthrough varicella, but we could not verify this hypothesis from the available data. For children aged $\geqslant 3$ years with only $\operatorname{Var}_{1}$ and no history of varicella disease, the visits for the 4 th oral polio vaccine at 4 years of age or the diphtheria and tetanus combined vaccine at 6 years of age may present an ideal time for $\operatorname{VarV}_{2}$ catch-up. Besides, a primary school-entry $\operatorname{VarV}_{2}$ requirement could be an efficient strategy to improve the coverage.

We did not find any difference in $\operatorname{VarV}_{1}$ and $\operatorname{VarV}_{2}$ coverage by children's gender, which was consistent with our previous study [9]. However, we found meaningful disparities in $\operatorname{VarV}_{1}$ and $\operatorname{VarV}_{2}$ coverage by children's immigration status. Lower coverage was observed in migrant children and it placed the migrant children at risk of varicella disease and of facilitating the transmission to other susceptible populations, as had been indicated for measles [10]. This result indicated that the utilization of vaccination services in migrant children was poorer than that in resident children, which could be associated with the lower demands for category II vaccines of the migrant caregivers and "service gap" that resulted from current allocation of public health resources. For migrant families, relative lower income and poor awareness of the importance of vaccination with category II vaccines are the main reasons for the lower demand for category II vaccines [9]. It means that a good income frees the household from the struggle of finding work to survive and would provide more spare time for caregivers to seek for public health service like immunization [11]. Besides, many of the migrant caregivers have a lower education level than residents, which results in a poorer understanding of the importance of vaccination of category II vaccines [11]. Furthermore, the public health resources, especially the vaccination nurses, are allocated according to the resident population with no consideration for the migrant population, which results in a "service gap". That means the vaccination service capacity is sometimes inadequate in areas where the volume of migrant children 
is large or dense. As we know, people believe that medical workers are an authoritative source of information and vaccination nurses can also make more precise recommendations on a case-by-case basis to children' caregivers when necessary. Unfortunately, most vaccination nurses working in vaccination clinics with a large scale migrant people do not have time to explain the importance of vaccinations to every caregivers. Therefore, the potential advantages of improving coverage posed through vaccination nurses' recommendations are not efficiently utilized in these areas. School-entry vaccination requirement, including regulation of 2-doses VarV, had succeeded in improving the coverage, irrespective of children's demographic characteristics such as the immigration status [12]. Therefore, the adoption and enforcement of a 2-doses VarV mandate in Zhejiang Province would not only be efficient in increasing the $\operatorname{VarV}_{2}$ coverage, but also address the coverage disparity between migrants and residents.

The coverage rates of VarV were significantly lower than those of MCV for both the 1st and the 2nd dose across all the birth cohorts. These findings were consistent with our previous survey conducted in Yiwu in 2014 [9] and demonstrates the importance of vaccines that are free of charge when setting for high coverage goals [13]. A higher VarV coverage could be expected if VarV were included in China's NIP as a category I vaccine. The proportion rates of children who received vaccination at the recommended age of $\operatorname{VarV}_{1}$ and $\operatorname{VarV}_{2}$ were increased rapidly over time. The main reason for this was the wide application of ZJIIS in the routine vaccination service in Zhejiang Province. ZJIIS allows the generation of alerts that reminded providers that a vaccination is due or overdue. ZJIIS makes the remind/recall strategy more feasible and efficient, and ensures up-to-date vaccination. Additionally, the parents also had the access to their children's vaccination records and information on the recommendation of vaccines via application programs derived from ZJIIS.

There are three limitations in our study. First, our analysis relied on the vaccination records of children registered in ZJIIS and the children who were not registered might have a lower coverage for all vaccines. As a result, we could have overestimated the coverage. Second, the ZJIIS data provides limited demographic information about the children and their families, so we could not explore other determinants of the coverage of VarV that were mentioned in the previous studies, such as caregivers' education level, or socio-economic status. Third, we could not correlate the coverage of VarV with varicella incidence since varicella was not presently a notifiable disease in China.

\section{Conclusions}

Our study showed a rapid increase in the $\operatorname{VarV}_{2}$ coverage, indicating a growing acceptance of the 2-doses VarV schedule by children's caregivers and physicians after the new recommendation was released. Further decline in the incidence of varicella disease can be expected as a higher 2-doses VarV vaccination coverage is achieved. Although our findings were encouraging, the coverage of VarV is still below the goal set for the NIP vaccine, placing school-age children at high risk of varicella or outbreaks. The findings of our study highlighted the necessity of school-entry VarV vaccination requirements to achieve high coverage $(>90 \%)$ and to eliminate disparities in coverage among subgroups. We also recommended that the coverage of VarV be continuously monitored via ZJIIS to identify any challenges to the current VarV vaccination program.

Acknowledgments: The authors would like to thank Rui Wu from Suzhou Shensu Automatic Technology Co., Ltd. for providing assistance with the ZJIIS data.

Author Contributions: Yu Hu and Qian Li conceived and designed the experiments; Yaping Chen performed the experiments; Bing Zhang and Yu Hu analyzed the data; Bing Zhang contributed reagents/materials/analysis tools; Yu Hu wrote the paper.

Conflicts of Interest: The authors declare no conflicts of interest.

\section{References}

1. Seward, J.F.; Marin, M.; Vazquez, M. Varicella vaccine effectiveness in the US vaccination program: A review. J. Infect. Dis. 2008, 197 (Suppl. 2), S82-S89. 
2. Kuter, B.; Matthews, H.; Shinefield, H.; Black, S.; Dennehy, P.; Watson, B.; Reisinger, K.; Kim, L.L.; Lupinacci, L.; Hartzel, J.; et al. Ten year follow-up of healthy children who received one or two injections of varicella vaccine. Pediatr. Infect. Dis. J. 2004, 23, 132-137. [CrossRef] [PubMed]

3. Li, Q.; Hu, Y.; Zhong, Y.; Chen, Y.; Tang, X.; Guo, J.; Shen, L. Using the Immunization Information System to determine vaccination coverage rates among children aged 1-7 years: A report from Zhejiang Province, China. Int. J. Environ. Res. Public Health 2014, 11, 2713-2728. [CrossRef] [PubMed]

4. Lopez, A.S.; Cardemil, C.; Pabst, L.J.; Cullen, K.A.; Leung, J.; Bialek, S.R. Two-dose varicella vaccination coverage among children aged 7 years-Six sentinel sites, United States, 2006-2012. Morb. Mortal. Wkly Rep. (MMWR) 2014, 63, 174-177. [PubMed]

5. $\quad$ Leung, J.; Lopez, A.S.; Blostein, J.; Thayer, N.; Zipprich, J.; Clayton, A.; Buttery, V.; Andersen, J.; Thomas, C.A.; Del Rosario, M.; et al. Impact of the US Two-dose Varicella Vaccination Program on the Epidemiology of Varicella Outbreaks: Data from Nine States, 2005-2012. Pediatr. Infect. Dis. J. 2015, 34, 1105-1109. [CrossRef] [PubMed]

6. Thomas, C.A.; Shwe, T.; Bixler, D.; del Rosario, M.; Grytdal, S.; Wang, C.; Haddy, L.E.; Bialek, S.R. Two-dose varicella vaccine effectiveness and rash severity in outbreaks of varicella among public school students. Pediatr. Infect. Dis. J. 2014, 33, 1164-1168. [CrossRef] [PubMed]

7. Chaves, S.S.; Gargiullo, P.; Zhang, J.X.; Civen, R.; Guris, D.; Mascola, L.; Seward, J.F. Loss of vaccine-induced immunity to varicella over time. N. Engl. J. Med. 2007, 356, 1121-1129. [CrossRef] [PubMed]

8. Seward, J.F.; Zhang, J.X.; Maupin, T.J.; Mascola, L.; Jumaan, A.O. Contagiousness of varicella in vaccinated cases: A household contact study. JAMA 2004, 292, 704-708. [CrossRef] [PubMed]

9. Hu, Y.; Luo, S.; Tang, X.; Lou, L.; Chen, Y.; Guo, J. Comparative assessment of immunization coverage of migrant children between national immunization program vaccines and non-national immunization program vaccines in East China. Hum. Vaccines Immunother. 2015, 11, 761-768. [CrossRef] [PubMed]

10. Santoli, J.M.; Setia, S.; Rodewald, L.E.; O'Mara, D.; Gallo, B.; Brink, E. Immunization pockets of need: Science and practice. Am. J. Prev. Med. 2000, 19 (Suppl. 3), 89-98. [CrossRef]

11. Hu, Y.; Li, Q.; Chen, E.; Chen, Y.; Qi, X. Determinants of childhood immunization uptake among socio-economically disadvantaged migrants in East China. Int. J. Environ. Res. Public Health 2013, 10, 2845-2856. [CrossRef] [PubMed]

12. Averhoff, F.; Linton, L.; Peddecord, K.M.; Edwards, C.; Wang, W.; Fishbein, D. A middle school immunization law rapidly and substantially increases immunization coverage among adolescents. Am. J. Public Health 2004, 94, 978-984. [CrossRef] [PubMed]

13. Blank, P.R.; Schwenkglenks, M.; Szucs, T.D. Vaccination coverage rates in eleven European countries during two consecutive influenza seasons. J. Infect. 2009, 58, 446-458. [CrossRef] [PubMed] 